\title{
The random case of Conley's theorem: II. The complete Lyapunov function*
}

\author{
Zhenxin Liu \\ College of Mathematics, and Key Laboratory of Symbolic Computation and Knowledge \\ Engineering of Ministry of Education, Jilin University, Changchun 130012, P.R. China \\ E-mail: zxliu@jlu.edu.cn
}

\begin{abstract}
Conley in [6] constructed a complete Lyapunov function for a flow on compact metric space which is constant on orbits in the chain recurrent set and is strictly decreasing on orbits outside the chain recurrent set. This indicates that the dynamical complexity focuses on the chain recurrent set and the dynamical behavior outside the chain recurrent set is quite simple. In this paper, a similar result is obtained for random dynamical systems under the assumption that the base space $(\Omega, \mathcal{F}, \mathbb{P})$ is a separable metric space endowed with a probability measure. By constructing a complete Lyapunov function, which is constant on orbits in the random chain recurrent set and is strictly decreasing on orbits outside the random chain recurrent set, the random case of Conley's fundamental theorem of dynamical systems is obtained. Furthermore, this result for random dynamical systems is generalized to noncompact state spaces.

Key words. Random dynamical systems; Random chain recurrent set; Complete Lyapunov function; Random local attractor; Fundamental theorem of dynamical systems Mathematics Subject Classification: 37H99, 37B35, 37B25, 37B55
\end{abstract}

\section{Introduction and main result}

In [6, Conley presented an important result in the study of dynamical systems-Conley decomposition theorem. This result, named after him, is also called "fundamental theorem of dynamical systems" [15], which can be stated very simply:

Theorem 1.1. (Conley's fundamental theorem of dynamical systems). Any flow on a compact metric space decomposes the space into a chain recurrent part and a gradientlike part.

The proof of the theorem relies on two important steps: the characterization of chain recurrent set in terms of attractors and the construction of complete Lyapunov function which provides the gradient-like structure for the theorem. To be more specific, assume $\left(X, d_{X}\right)$ is a compact metric space and $\varphi$ is a flow on $X$. For given $\epsilon, T>0$, a finite sequence $\left(x_{0}, t_{0}\right),\left(x_{1}, t_{1}\right), \cdots,\left(x_{n}, t_{n}\right)$ in $X \times(0, \infty)$ is called an $\epsilon$-T-chain for $\varphi$ if

$$
d_{X}\left(\varphi\left(t_{j}, x_{j}\right), x_{j+1}\right)<\epsilon, t_{j} \geq T
$$

\footnotetext{
*Published in: Nonlinearity 20 (2007), 1017-1030.
} 
for $j=0,1, \cdots, n-1$. Here $n$ is called the length of the chain. A point $p \in X$ is called chain recurrent if for any $\epsilon, T>0$, there is an $\epsilon$-T-chain with the length at least 1 which begins and ends at $p$. The set of all chain recurrent points, denoted by $\mathcal{C R}(\varphi)$, is called the chain recurrent set of $\varphi$. Conley [6] showed that the chain recurrent set $\mathcal{C R}(\varphi)$ equals the intersection of the union of attractor-repeller pair $(A, R)$ as $A$ varies over the collection of attractors of $\varphi$, i.e.

$$
\mathcal{C R}(\varphi)=\bigcap[A \cup R]
$$

Based on this characterization, he constructed a complete Lyapunov function. A complete Lyapunov function for $\varphi$ on $X$ is a continuous, real-valued function $L$ with properties: (1) it is strictly decreasing on orbits outside the chain recurrent set while is constant on orbits in the chain recurrent set; $(2) L(\mathcal{C R}(\varphi))$ is nowhere dense; and (3) it separates different components of the chain recurrent set. A dynamical system is called gradient-like if there exists some continuous real-valued function which is strictly decreasing on nonconstant solutions, see [6] for details of these definitions and some interesting examples.

Conley's result was adapted for maps on compact spaces by Franks [9], was later established for maps on locally compact metric spaces by Hurley [10, 11, and was extended by Hurley [12 for semiflows and maps on arbitrary metric spaces. To extend Conley decomposition theorem to random dynamical systems (RDS), Liu [13] introduced the definitions of random chain recurrent set and appropriate random attractor. He then took the first step towards the random case of Conley decomposition theorem-the characterization of random chain recurrent set in terms of random attractors. In this paper, we intend to complete the second step-constructing a complete Lyapunov function, which provides the gradient-like structure for RDS. Here, "random gradient-like" could be understood in this way: the skew-product flow $\Theta_{t}(\omega, x):=\left(\theta_{t} \omega, \varphi(t, \omega) x\right)$ corresponding to $\operatorname{RDS} \varphi$ (see [1, 2, 5] for details) flows downhill with respect to a function (i.e. complete Lyapunov function) along non-stationary solutions (a solution $u(\omega)$ is called stationary if $\left.\varphi(t, \omega) u(\omega)=u\left(\theta_{t} \omega\right)\right)$. The main result of this paper states as follows:

Theorem 1.2. Assume $(\Omega, \mathcal{F}, \mathbb{P})$ is a separable metric space endowed with a probability measure, where $\mathcal{F}=\mathcal{B}(\Omega)$, the Borel $\sigma$-algebra on $\Omega$. Let $X$ be a compact metric space, and $\varphi$ be an $R D S$ with the base space $(\Omega, \mathcal{F}, \mathbb{P})$ and the state space $X$, then there exists a complete Lyapunov function for $\varphi$ (defined in Definition 4.1) on $X$.

By the main theorem in [13] and Theorem 1.2 above we arrive at the random case of Conley decomposition theorem:

Theorem 1.3. (random Conley decomposition theorem). Any random dynamical system (with a separable metric space endowed with a probability measure as base space) on a compact metric space decomposes the space into a random chain recurrent part and a random gradient-like part.

\section{Preliminaries}

In this section two preliminary definitions are given. For other definitions, notations and preliminary propositions we refer the readers to [13] for details.

Definition 2.1. Let $X$ be a metric space with a metric $d_{X}$. A set-valued map $\omega \mapsto D(\omega)$ taking values in the closed/compact subsets of $X$ is said to be a random closed/compact set if the mapping $\omega \mapsto \operatorname{dist}_{X}(x, D(\omega))$ is measurable for any $x \in X$, where $\operatorname{dist}_{X}(x, B):=$ 
$\inf _{y \in B} d_{X}(x, y)$. A set-valued map $\omega \mapsto U(\omega)$ taking values in the open subsets of $X$ is said to be a random open set if $\omega \mapsto U^{c}(\omega)$ is a random closed set, where $U^{c}$ denotes the complement of $U$.

Definition 2.2. For any given random set $D(\omega)$, we denote $\Omega_{D}(\omega)$ the omega-limit set of $D(\omega)$, which is determined as follows:

$$
\Omega_{D}(\omega):=\bigcap_{T \geq 0} \overline{\bigcup_{t \geq T} \phi\left(t, \theta_{-t} \omega\right) D\left(\theta_{-t} \omega\right)}
$$

and we denote $\alpha_{D}(\omega)$ the alpha-limit set of $D(\omega)$, which is determined as follows:

$$
\alpha_{D}(\omega):=\bigcap_{T \geq 0} \overline{\bigcup_{t \geq T} \phi\left(-t, \theta_{t} \omega\right) D\left(\theta_{t} \omega\right)}
$$

In Sections 2-4, we will always assume that $X$ is a compact metric space, therefore it is a Polish space, i.e. a separable complete metric space.

Remark 2.1. Assume $U(\omega)$ is a random pre-attractor and $A(\omega)$ is the random local attractor inside $U(\omega)$ (see [13] for the definitions), then it is easy to see that

$$
\begin{aligned}
A(\omega) & =\bigcap_{n \in \mathbb{N}} \overline{\bigcup_{s \geq n T(\omega)} \varphi\left(s, \theta_{-s} \omega\right) U\left(\theta_{-s} \omega\right)} \\
& =\bigcap_{t \geq T(\omega)} \overline{\bigcup_{s \geq t} \varphi\left(s, \theta_{-s} \omega\right) U\left(\theta_{-s} \omega\right)} \\
& =\bigcap_{t \geq 0} \overline{\bigcup_{s \geq t}\left(s, \theta_{-s} \omega\right) U\left(\theta_{-s} \omega\right)} \\
& =\Omega_{U}(\omega) .
\end{aligned}
$$

Conversely, if a random open set $U(\omega)$ satisfies $\Omega_{U}(\omega) \subset U(\omega)$, we define

$$
T(\omega):=\inf \left\{t \in \mathbb{R}^{+} \mid \varphi\left(t, \theta_{-t} \omega\right) \overline{U\left(\theta_{-t} \omega\right)} \subset V(\omega)\right\},
$$

where $V(\omega) \subset U(\omega)$ is a closed neighborhood of $\Omega_{U}(\omega)$. Then, by a similar argument to the proof of Lemma 3.5 in [13, we obtain that $T(\omega)$ is measurable. Hence $U(\omega)$ is a random pre-attractor and $\Omega_{U}(\omega)$ is the random attractor determined by $U(\omega)$. That is, an invariant random compact set is an attractor if and only if it is the omega-limit set of one of its random neighborhoods.

\section{Improvement of main result in [13]}

Lemma 3.1. Assume $U(\omega)$ is a random open set and an invariant random compact set $A(\omega) \subset U(\omega)$ satisfies that $\Omega_{U}(\omega)=A(\omega)$, then there exists a forward invariant random open set $\tilde{U}(\omega)$ with the same properties as $U(\omega)$.

Proof. Let

$$
\tilde{U}(\omega):=\operatorname{int}\left[\overline{\bigcup_{t \geq 0} \varphi\left(t, \theta_{-t} \omega\right) U\left(\theta_{-t} \omega\right)}\right]
$$


then by Proposition 1.5.1 of [5], $\tilde{U}(\omega)$ is a forward invariant random open set and $A(\omega) \subset$ $\tilde{U}(\omega)$ (note that $U(\omega) \subset \tilde{U}(\omega)$ ). Now we show $\Omega_{\tilde{U}}(\omega)=A(\omega)$.

$$
\begin{aligned}
\Omega_{\tilde{U}}(\omega) & =\bigcap_{T \geq 0} \overline{\bigcup_{s \geq T} \varphi\left(s, \theta_{-s} \omega\right) \tilde{U}\left(\theta_{-s} \omega\right)} \\
& =\bigcap_{T \geq 0} \overline{\bigcup_{s \geq T}\left[\varphi\left(s, \theta_{-s} \omega\right) \bigcup_{t \geq 0} \varphi\left(t, \theta_{-t} \circ \theta_{-s} \omega\right) U\left(\theta_{-t} \circ \theta_{-s} \omega\right)\right]} \\
& =\bigcap_{T \geq 0} \overline{\bigcup_{s \geq T} \varphi\left(s, \theta_{-s} \omega\right) \circ \varphi\left(t, \theta_{-t} \circ \theta_{-s} \omega\right) U\left(\theta_{-t} \circ \theta_{-s} \omega\right)} \\
& =\bigcap_{T \geq 0} \overline{\bigcup_{s \geq T} \varphi\left(s+t, \theta_{-s-t} \omega\right) U\left(\theta_{-s-t} \omega\right)} \\
& =\bigcap_{T \geq 0} \overline{\bigcup_{s \geq T} \varphi\left(s, \theta_{-s} \omega\right) U\left(\theta_{-s} \omega\right)} \\
& =\Omega_{U}(\omega)=A(\omega),
\end{aligned}
$$

where (11) holds because for any random set $D(\omega)$ we have

$$
\overline{\bigcup_{t \geq T} \varphi\left(t, \theta_{-t} \omega\right) D\left(\theta_{-t} \omega\right)}=\overline{\bigcup_{t \geq T} \varphi\left(t, \theta_{-t} \omega\right) \overline{D\left(\theta_{-t} \omega\right)}}
$$

This completes the proof.

In contrast to Lemma 3.3 in [13, we have the following finer result.

Corollary 3.1. For arbitrary random local attractor $A(\omega)$, the random basin $B(A)(\omega)$ determined by $A(\omega)$ is an invariant random open set.

Proof. According to Lemma3.1 and Remark 2.1, without loss of generality, we can assume that a forward invariant random open set $U(\omega)$ is a random pre-attractor corresponding to the attractor $A(\omega)$. Hence by Lemma 4.2 of [7] the corollary follows.

Remark 3.1. (i) By Lemma 3.1 and Remark 2.1 we know that, for arbitrary random attractor $A(\omega)$, there exists a forward invariant random pre-attractor $U(\omega)$ which determines $A(\omega)$. Moreover, by the fact $\Omega_{U}(\omega)=A(\omega)$, it follows that $A(\omega)$ pull-back attracts $\overline{U(\omega)}$. Hence it pull-back attracts any random closed set inside $U(\omega)$. The random attractor defined in [7] only weakly attracts (i.e. attracts in probability) all random closed sets inside its fundamental neighborhood. Therefore, the random attractor we defined in [13. (hence in present paper) is stronger than that of [7]. On the other hand, the attractor defined in [13] and [14] are equivalent. In fact, it is clear that the interior of fundamental neighborhood [14] plays the role of random pre-attractor [13]; conversely, the closure of random pre-attractor plays the role of fundamental neighborhood.

(ii) For a given random local attractor $A(\omega)$, we can define the random repeller corresponding to $A(\omega)$ to be $R(\omega):=X-B(A)(\omega)$ similar to that in [7, 14] and we call the pair $(A, R)$ a random attractor-repeller pair. In fact, $R(\omega)$ is a random repeller with respect to its random neighborhood $X-\overline{U(\omega)}$, see [14] or forthcoming Lemma 5.1 for the proof. As for the properties of random attractor-repeller pair, the reader can refer to [7, 14] for details.

Therefore, the main theorem in [13] can be restated in the following theorem, which characterizes random chain recurrent set using its original form in the deterministic case. 
Theorem 3.1. Assume $X$ is a compact metric space, $A(\omega)$ is a random local attractor and $R(\omega)$ is the random repeller corresponding to $A(\omega)$, then

$$
\mathcal{C R}_{\varphi}(\omega)=\bigcap[A(\omega) \cup R(\omega)]
$$

almost surely, where the intersection is taken over all random local attractors.

\section{Complete Lyapunov function}

Lemma 4.1. Assume $(A, R)$ is an attractor-repeller pair of $\varphi$, then there exists an $\mathcal{F} \times$ $\mathcal{B}(X)$-measurable Lyapunov function l for $(A, R)$ such that:

(i) $l(\omega, x)=0$ when $x \in A(\omega)$, and $l(\omega, x)=1$ when $x \in R(\omega)$;

(ii) for $\forall x \in X \backslash(A(\omega) \cup R(\omega))$ and $\forall t>0,1>l(\omega, x)>l\left(\theta_{t} \omega, \varphi(t, \omega) x\right)>0$.

Proof. The idea of the proof is originated from [3, 2]. Assume $U(\omega)$ is a forward invariant random pre-attractor of $A(\omega)$, and we define the first entrance time of $\varphi(t, \omega) x$ into $\overline{U\left(\theta_{t} \omega\right)}$ as follows:

$$
\tau(\omega, x):= \begin{cases}-\infty, & x \in A(\omega) \\ \inf \left\{t \in \mathbb{R} \mid \varphi(t, \omega) x \in \overline{U\left(\theta_{t} \omega\right)}\right\}, & x \in X \backslash(A(\omega) \cup R(\omega)) ; \\ +\infty, & x \in R(\omega) .\end{cases}
$$

Since $\omega \mapsto d(x, \overline{U(\omega)})$ is measurable, $x \mapsto d(x, \overline{U(\omega)})$ is continuous, we have that $(\omega, x) \mapsto d(x, \overline{U(\omega)})$ is measurable. Hence for arbitrary $t \in \mathbb{R},(\omega, x) \mapsto d\left(\varphi(t, \omega) x, \overline{U\left(\theta_{t} \omega\right)}\right)$ is measurable. For $\forall a \in \mathbb{R}$, we have

$$
\{(\omega, x) \mid \tau(\omega, x) \geq a\}=\bigcap_{t<a, t \in \mathbb{Q}}\left\{(\omega, x) \mid d\left(\varphi(t, \omega) x, \overline{U\left(\theta_{t} \omega\right)}\right)>0\right\}
$$

which verifies that $(\omega, x) \mapsto \tau(\omega, x)$ is measurable.

By the definition of $\tau(\omega, x)$ in (2), we have

$$
\begin{aligned}
\tau\left(\theta_{t} \omega, \varphi(t, \omega) x\right) & =\inf \left\{s \in \mathbb{R} \mid \varphi\left(s, \theta_{t} \omega\right) \circ \varphi(t, \omega) x \in \overline{U\left(\theta_{t+s} \omega\right)}\right\} \\
& =\inf \left\{s \in \mathbb{R} \mid \varphi(t+s, \omega) x \in \overline{U\left(\theta_{t+s} \omega\right)}\right\} \\
& =\tau(\omega, x)-t
\end{aligned}
$$

Define

$$
l(\omega, x)= \begin{cases}\frac{1}{2} \mathrm{e}^{\tau(\omega, x)} & -\infty \leq \tau(\omega, x)<0 \\ \frac{1}{2}\left(1+\frac{2}{\pi} \arctan \tau(\omega, x)\right), & 0 \leq \tau(\omega, x) \leq+\infty\end{cases}
$$

Since $\tau(\omega, x)$ is $\mathcal{F} \times \mathcal{B}(X)$-measurable, hence $l(\omega, x)$ is. It is obvious that the so defined $l(\omega, x)$ satisfies (i), while (ii) follows from the fact $\tau\left(\theta_{t} \omega, \varphi(t, \omega) x\right)=\tau(\omega, x)-t$. This terminates the proof of the lemma.

Denote $\tilde{\mathcal{M}}=\{x \mid x: \Omega \mapsto X$ measurable $\}$. For any $x(\omega), y(\omega) \in \tilde{\mathcal{M}}$, define

$$
d_{\tilde{\mathcal{M}}}(x, y)=\int_{\Omega} d_{X}(x(\omega), y(\omega)) \mathrm{d} \omega
$$

It is clear that $d_{\tilde{\mathcal{M}}}(\cdot, \cdot)$ is a metric on $\tilde{\mathcal{M}}$. By the separability of $\Omega$ and $X$, we can assume that $\left\{U_{i}\right\}_{i=1}^{\infty}$ is a countable basis for the topology of $\Omega$, and $\left\{x_{j}\right\}_{j=1}^{\infty}$ are countable dense 
subsets of $X$. For $U_{i}, i=1, \cdots, n$ with $U_{i} \cap U_{j}=\emptyset, i \neq j$ and $x_{k}, k=1, \cdots, n+1$, we define the following step-like function:

$$
\chi_{U_{1}, \cdots, U_{n}}\left(x_{1}, \cdots, x_{n+1}\right)= \begin{cases}x_{1}, & \omega \in U_{1}, \\ \cdots & \cdots \\ x_{n}, & \omega \in U_{n}, \\ x_{n+1}, & \omega \in \Omega-\bigcup_{i=1}^{n} U_{i} .\end{cases}
$$

Then it is easy to see that the step-like functions defined above are countable and they constitute a countable dense subset of $\tilde{\mathcal{M}}$. For definiteness, we denote this countable dense subset of $\tilde{\mathcal{M}}$ as $\mathcal{M}=\left\{x_{n}(\omega)\right\}_{n \in \mathbb{N}}$. Similarly, denote $\mathcal{N}$ the countable dense subset of $\tilde{\mathcal{N}}$, the set of all measurable maps from $\Omega$ to $\mathbb{R}^{+}$. By the proof of Lemma 3.7 in [13], for any given $x(\omega) \in \mathcal{M}, \epsilon(\omega), T(\omega) \in \mathcal{N}$, we can obtain a random pre-attractor as expressed by (3.11) in [13, and we denote it here by $U(x, \epsilon, T)$. Therefore we can obtain a random local attractor $A(x, \epsilon, T)$ determined by $U(x, \epsilon, T)$. Note that the random local attractors obtained in this way are countable, and we denote all of them by $\mathcal{A}$.

Lemma 4.2. Assume $(\Omega, \mathcal{F}, \mathbb{P})$ is a separable metric space endowed with a probability measure, where $\mathcal{F}=\mathcal{B}(\Omega)$, then

$$
\mathcal{C R}_{\varphi}(\omega)=\bigcap_{A \in \mathcal{A}}[A(\omega) \cup R(\omega)]
$$

almost surely, where $\mathcal{A}$ is defined as above.

Proof. By Theorem 3.1 we only need to prove the right hand side of (3) is contained in the left hand side. Note that $\mathcal{N}, \mathcal{M}$ is dense in $\tilde{\mathcal{N}}, \tilde{\mathcal{M}}$, respectively. The remaining proof is similar to that of Lemma 3.7 in $[13$, here we omit the details.

Definition 4.1. A complete Lyapunov function for the $R D S \varphi$ is an $\mathcal{F} \times \mathcal{B}(X)$-measurable function $L: \Omega \times X \mapsto \mathbb{R}^{+}$with the following properties:

(1) $L\left(\theta_{t} \omega, \varphi(t, \omega) x\right)=L(\omega, x), \forall t>0$ when $x \in \mathcal{C R}_{\varphi}(\omega)$;

(2) If $x \in X-\mathcal{C R}_{\varphi}(\omega)$, then

$$
L\left(\theta_{t} \omega, \varphi(t, \omega) x\right)<L(\omega, x), \forall t>0
$$

(3) The range of $L$ on $\mathcal{C R}_{\varphi}(\omega)$ is a compact nowhere dense subset of $[0,1]$;

(4) $L$ separates different random chain transitive components of $\varphi$ (to be defined below).

For definiteness, denote the above $\mathcal{A}=\left\{A_{n}(\omega) \mid n \in \mathbb{N}\right\}$ and assume $l_{n}(\omega, x)$ is the Lyapunov function determined by the random attractor-repeller $\left(A_{n}, R_{n}\right)$ in Lemma 4.1 . Define

$$
L(\omega, x)=\sum_{n=1}^{\infty} \frac{2 l_{n}(\omega, x)}{3^{n}} .
$$

Later we will show that $L(\omega, x)$ defined by (4) is a complete Lyapunov function for $\varphi$. Firstly it is easy to see that $L(\omega, x)$ is well defined since the sum in (44) is uniformly convergent and $L(\omega, x)$ is $\mathcal{F} \times \mathcal{B}(X)$-measurable since each $l_{n}(\omega, x)$ is.

A critical value of $L$ is defined to be one achieved on the random chain recurrent set. For any $A(\omega) \in \mathcal{A}$, we have $\mathcal{C R}_{\varphi}(\omega) \subset A(\omega) \cup R(\omega)$ by Lemma 4.2. Hence each $l_{n}(\omega, x)$ is either 0 or 1 at a point of the random chain recurrent set. Therefore the set of the critical 
values of $L$ is a compact nowhere dense subset of $[0,1]$. For a given critical value $c$ of $L$, denote $L^{-1}(c)=\Omega \times\{\tilde{C}(\omega)\}$. Then by the measurability of $L$, the graph of $\tilde{C}(\omega)$

$$
\operatorname{graph}(\tilde{C})=\{(\omega, x) \mid x \in \tilde{C}(\omega)\}=\{(\omega, x) \mid L(\omega, x)=c\}
$$

is $\mathcal{F} \times \mathcal{B}(X)$-measurable. Hence $\tilde{C}(\omega)$ is an $\mathcal{F}^{u}$-measurable random closed set by Theorem III.30 on page 80 of [4]. Denote $C(\omega)=\tilde{C}(\omega) \cap \mathcal{C R}_{\varphi}(\omega)$, then $C(\omega)$ is an $\mathcal{F}^{u}$-measurable random compact set (since $\mathcal{C} \mathcal{R}_{\varphi}(\omega)$ is an $\mathcal{F}^{u}$-measurable random compact set). For convenience, we also use $L^{-1}(c) \cap \mathcal{C} \mathcal{R}_{\varphi}(\omega)$ to denote $C(\omega)$, indicating that $C(\omega)$ is determined by the critical value $c$ of $L$. Since $C(\omega)$ is determined by the critical value $c$, we have

$$
C(\omega) \subset A(\omega) \mathbb{P} \text {-a.s. or } C(\omega) \subset R(\omega) \mathbb{P} \text {-a.s. }
$$

for any $A(\omega) \in \mathcal{A}$, where $(A, R)$ is a random attractor-repeller pair.

We call $C(\omega)$ obtained in this way a random chain transitive component of $\varphi$. By Lemma 4.2 we have that a chain transitive component $C(\omega)$ can be expressed by

$$
C(\omega)=\bigcap_{n \in \mathbb{N}} C_{n}(\omega)
$$

where $C_{n}(\omega)=A_{n}(\omega)$ or $C_{n}(\omega)=R_{n}(\omega)$, recalling that we have assumed that $\mathcal{A}=$ $\left\{A_{n}(\omega) \mid n \in \mathbb{N}\right\}$.

Definition 4.2. Assume $C, C^{\prime}$ are distinct random chain transitive components of $\varphi$. We say that a random local attractor $A(\omega)$ distinguishes between them if either $C(\omega) \subset A(\omega)$ and $C^{\prime}(\omega) \subset R(\omega)$ or if the corresponding conditions hold with $C$ and $C^{\prime}$ interchanged.

An equivalence relation on the set of random chain recurrent variables is given by: $x(\omega) \sim y(\omega)$ if and only if for arbitrary $\epsilon(\omega), T(\omega)>0$, there is one $\epsilon(\omega)$-T( $\omega)$-chain from $x(\omega)$ to $y(\omega)$ and another one from $y(\omega)$ to $x(\omega) \mathbb{P}$-a.s.

With respect to the relation between random chain transitive component of $\varphi$ and the equivalence relation introduced above, we have the following proposition:

Proposition 4.1. (1) Assume $C(\omega)$ is a random chain transitive component of $\varphi$, then for $\forall x(\omega), y(\omega) \in C(\omega)$, we have $x(\omega) \sim y(\omega)$.

(2) Assume $C^{\prime}(\omega)$ is another random chain transitive component of $\varphi$, then for $\forall x(\omega) \in$ $C(\omega)$ and $\forall z(\omega) \in C^{\prime}(\omega)$, we have $x(\omega) \not z(\omega)$.

Proof. (1) We only need to prove that for $\forall \epsilon(\omega), T(\omega) \in \mathcal{N}$, there exist $\epsilon(\omega)-T(\omega)$ chains from $x(\omega)$ to $y(\omega)$ and from $y(\omega)$ to $x(\omega) \mathbb{P}$-a.s. If this is false, say, there exist $\epsilon_{0}(\omega), T_{0}(\omega) \in \mathcal{N}$ such that there is no $2 \epsilon_{0}-T_{0}$-chain from $x(\omega)$ to $y(\omega) \mathbb{P}$-a.s. By the density of $\mathcal{M}$ in $\tilde{\mathcal{M}}$, there exists some $x_{1}(\omega) \in \mathcal{M}$ such that $x(\omega) \in B\left(A\left(x_{1}, \epsilon_{0}, T_{0}\right)\right) \mathbb{P}$-a.s. Since $x(\omega)$ is random chain recurrent, we have $x(\omega) \in A\left(x_{1}, \epsilon_{0}, T_{0}\right) \mathbb{P}$-a.s. by Lemma 3.6 of [13]. Since there is no $2 \epsilon_{0}-T_{0}$-chain from $x(\omega)$ to $y(\omega) \mathbb{P}$-a.s., we can obtain that $y(\omega) \notin A\left(x_{1}, \epsilon_{0}, T_{0}\right)$ with positive probability. In fact, if $y(\omega) \in A\left(x_{1}, \epsilon_{0}, T_{0}\right) \mathbb{P}$-a.s., then we have $y(\omega) \in A\left(x_{1}, \epsilon_{0}, T_{0}\right) \subset U\left(x, 2 \epsilon_{0}, T_{0}\right) \mathbb{P}$-a.s. when $x_{1}(\omega)$ is close enough to $x(\omega)$. Hence we obtain that there is $2 \epsilon_{0}-T_{0}$-chain from $x(\omega)$ to $y(\omega) \mathbb{P}$-a.s. by the definition of $U\left(x, 2 \epsilon_{0}, T_{0}\right)$, a contradiction.

(2) To see this, notice that

$$
\mathcal{C R}_{\varphi}(\omega)=\bigcup_{c \text { critical value }}\left[L^{-1}(c) \cap \mathcal{C} \mathcal{R}_{\varphi}(\omega)\right] .
$$


Assume that the two random transitive components $C(\omega)$ and $C^{\prime}(\omega)$ are determined by two critical values $c \neq c^{\prime}$ of $L$, respectively. That is

$$
C(\omega)=L^{-1}(c) \cap \mathcal{C} \mathcal{R}_{\varphi}(\omega), \quad C^{\prime}(\omega)=L^{-1}\left(c^{\prime}\right) \cap \mathcal{C} \mathcal{R}_{\varphi}(\omega) .
$$

Since $c \neq c^{\prime}$, there exists some $n_{0} \in \mathbb{N}$ such that $l_{n_{0}}$ is 0 on $C(\omega)$ and is 1 on $C^{\prime}(\omega)$ or the converse holds. Hence we have

$$
C(\omega) \subset A_{n_{0}}(\omega), \quad C^{\prime}(\omega) \subset R_{n_{0}}(\omega) \mathbb{P} \text {-a.s. }
$$

or

$$
C^{\prime}(\omega) \subset A_{n_{0}}(\omega), \quad C(\omega) \subset R_{n_{0}}(\omega) \mathbb{P} \text {-a.s. }
$$

Without loss of generality, we assume the former case holds. If for any $\epsilon(\omega), T(\omega)>0$, there exists an $\epsilon(\omega)-T(\omega)$-chain from $x(\omega)$ to $z(\omega) \mathbb{P}$-a.s. Then by the proof of Lemma 3.4 of [13], we obtain that $z(\omega) \in A_{n_{0}}(\omega) \mathbb{P}$-a.s., a contradiction to $z(\omega) \in C^{\prime}(\omega)$. That is $x(\omega) \nsim z(\omega)$.

Remark 4.1. By the above proof we know that if $C, C^{\prime}$ are distinct random chain transitive components of $\varphi$, then there exists an element $A(\omega) \in \mathcal{A}$ that distinguishes between them.

Lemma 4.3. Assume $x(\omega), y(\omega) \in \mathcal{C R}_{\varphi}(\omega) \mathbb{P}$-a.s. with the property that for $\forall \epsilon(\omega)$, $T(\omega)>0$ there is a random $\epsilon(\omega)-T(\omega)$-chain from $x(\omega)$ to $y(\omega) \mathbb{P}$-a.s. If $A(\omega)$ is a random local attractor containing $x(\omega) \mathbb{P}$-a.s., then $A(\omega)$ also contains $y(\omega) \mathbb{P}$-a.s.

Proof. If $y(\omega) \notin A(\omega)$ with positive probability, then by Lemma 3.6 of [13] we have $y(\omega) \in X-B(A)(\omega)$ with positive probability. Since for $\forall \epsilon(\omega), T(\omega)>0$, there is a random $\epsilon(\omega)-T(\omega)$-chain from $x(\omega)$ to $y(\omega) \mathbb{P}$-a.s., similar to the proof of Lemma 3.4 of [13] and by the fact $x(\omega) \in A(\omega)$, we obtain that $y(\omega) \in U(\omega) \subset B(A)(\omega) \mathbb{P}$-a.s., where $U(\omega)$ is a random pre-attractor which determines $A(\omega)$, a contradiction.

Corollary 4.1. Assume $C, C^{\prime}$ are two random chain transitive components of $\varphi$ with the property that for $\forall \epsilon(\omega), T(\omega)>0$ there is a random $\epsilon(\omega)-T(\omega)$-chain from $C$ to $C^{\prime} \mathbb{P}$-a.s. If $A(\omega)$ is a random local attractor containing $C \mathbb{P}$-a.s., then $A(\omega)$ also contains $C^{\prime} \mathbb{P}$-a.s.

Proof. The corollary follows directly from Lemma 4.3.

Now we are ready to prove our main result-Theorem 1.2

Theorem 4.1. (complete Lyapunov function). The function defined by (4) is a complete Lyapunov function for the $R D S \varphi$. Moreover, if $C$ and $C^{\prime}$ are distinct random chain transitive components of $\varphi$ with the property that for arbitrary $\epsilon(\omega), T(\omega)>0$ there is an $\epsilon(\omega)-T(\omega)$-chain from $C$ to $C^{\prime} \mathbb{P}$-a.s., then $L(\Omega, C)>L\left(\Omega, C^{\prime}\right)$.

Proof. We first verify (1)-(4) of Definition 4.1 one by one.

(1): This follows from the fact that if $x \in \mathcal{C R}_{\varphi}(\omega)$, then

$$
l_{n}(\omega, x)=l_{n}\left(\theta_{t} \omega, \varphi(t, \omega) x\right), \forall t>0
$$

takes value 0 or 1 for each $n \in \mathbb{N}$.

(2): Since $x \in X-\mathcal{C} \mathcal{R}_{\varphi}(\omega)$, there exists an $A_{n} \in \mathcal{A}$ such that $x \in X-A_{n}(\omega) \cup R_{n}(\omega)$. Then by Lemma 4.1 we have

$$
l_{n}\left(\theta_{t} \omega, \varphi(t, \omega) x\right)<l_{n}(\omega, x), \forall t>0 .
$$


(3): It has been proved above.

(4): By the definition of random chain transitive component, it is clear that $L$ is constant on each random chain transitive component and $L$ takes different values on different random chain transitive components. This verifies (4).

By Corollary 4.1, any random local attractor containing $C$ must also contain $C^{\prime}$, therefore $l_{n}=0$ on $C$ implies $l_{n}=0$ on $C^{\prime}$, that is $l_{n}(\Omega, C) \geq l_{n}\left(\Omega, C^{\prime}\right)$ for each $n \in \mathbb{N}$. Hence we have obtained $L(\Omega, C) \geq L\left(\Omega, C^{\prime}\right)$, this together with (4) verifies $L(\Omega, C)>L\left(\Omega, C^{\prime}\right)$.

It should be pointed out that random chain transitive components are not determined by the complete Lyapunov function, but by the RDS $\varphi$ itself. See (6).

Now we give two simple examples to illustrate our results.

Example 4.1. (1) In Example 4.1 of [13, we have shown that $\mathcal{C} \mathcal{R}_{\varphi}(\omega)=\{-1,0,1\} \mathbb{P}$-a.s. By Theorem 1.2 we can construct a complete Lyapunov function for $\varphi$. It is clear that $C_{1}=\{-1\}, C_{2}=\{0\}, C_{3}=\{1\}$ are three random chain transitive components of $\varphi$. Here $W_{t}$ is a Wiener process for which we take two-sided time $t \in \mathbb{R}$. This is standard to put a stochastic differential equation in the framework of RDS, see [1, 2, 5, 8, etc for details.

(2) In Example 4.2 of [13, we have shown that the $\operatorname{RDS} \varphi$ has no non-trivial attractor besides $X$ and $\emptyset$. Hence $\mathcal{C R}_{\varphi}(\omega)=X \mathbb{P}$-a.s. Therefore, by (6) we obtain that the only random chain transitive component is $X$, i.e. the random chain recurrent set itself.

\section{$5 \quad$ Extension to noncompact spaces}

We know that assuming $X$ being compact is too restrictive for applications, so in this section we assume that $X$ is a Polish space and try to extend Conley decomposition theorem for RDS to noncompact Polish spaces.

Definition 5.1. Assume $\epsilon(\omega)>0$ is a random variable.

(i) A random open set $U(\omega)$ is called $\epsilon$-absorbing if there exists a random variable $T(\omega)>0$ such that $U(\omega)$ contains the $\epsilon$-neighborhood of $U_{T}(\omega):=\overline{\bigcup_{t \geq T} \varphi\left(t, \theta_{-t} \omega\right) U\left(\theta_{-t} \omega\right)}$, i.e.

$$
B_{\epsilon}\left(U_{T}(\omega)\right) \subset U(\omega) .
$$

And we call a random open set $U(\omega)$ absorbing if it is $\epsilon$-absorbing for some random variable $\epsilon(\omega)>0$.

(ii) A random open set $V(\omega)$ is called $\epsilon$-repelling if there exists a random variable $T(\omega)>0$ such that $V(\omega)$ contains the $\epsilon$-neighborhood of $\hat{V}_{T}(\omega):=\overline{\bigcup_{t \geq T} \varphi\left(-t, \theta_{t} \omega\right) V\left(\theta_{t} \omega\right)}$, i.e.

$$
B_{\epsilon}\left(\hat{V}_{T}(\omega)\right) \subset V(\omega) .
$$

And we call a random open set $V(\omega)$ repelling if it is $\epsilon$-repelling for some random variable $\epsilon(\omega)>0$.

Definition 5.2. (i) An invariant random closed set $A(\omega)$ is called an (local) attractor if there exists an absorbing neighborhood $U(\omega)$ of $A(\omega)$ such that $A(\omega)=\Omega_{U}(\omega)$. And we call

$$
B(A, U)(\omega):=\left\{x \mid \varphi(t, \omega) x \in U\left(\theta_{t} \omega\right) \text { for some } t \geq 0\right\}
$$

the basin of attraction of $A(\omega)$ with respect to $U(\omega)$.

(ii) An invariant random closed set $R(\omega)$ is called a (local) repeller if there exists a repelling neighborhood $V(\omega)$ of $R(\omega)$ such that $R(\omega)=\alpha_{U}(\omega)$. And we call

$$
B(R, V)(\omega):=\left\{x \mid \varphi(t, \omega) x \in V\left(\theta_{t} \omega\right) \text { for some } t \leq 0\right\}
$$


the basin of repulsion of $R(\omega)$ with respect to $V(\omega)$.

Remark 5.1. (i) When $X$ is compact, the basin of attraction of an attractor is independent of the choice of absorbing neighborhoods; but when $X$ is not compact, the basin is generally dependent on absorbing neighborhoods. Of course the same conclusion holds for basin of repulsion. See [10] for details.

(ii) For any random set $D(\omega), \Omega_{D}(\omega)$ is invariant even if $X$ loses compactness. See Lemma 3.2 and Remark 3.7 of [8] for details. Similarly, the alpha-limit set $\alpha_{D}(\omega)$ is also invariant.

Lemma 5.1. Assume $\varphi$ is an $R D S$ on a Polish space $X$, and assume $A(\omega)$ is an attractor of $\varphi$ with a forward invariant absorbing neighborhood $U(\omega)$ and the basin of attraction with respect to $U, B(A, U)(\omega)$. Then $R(\omega):=X-B(A, U)(\omega)$ is a random repeller with a repelling neighborhood $V(\omega):=X-\overline{U(\omega)}$ and the basin of repulsion with respect to $V(\omega)$ being $B(R, V)(\omega)=X-A(\omega)$. (We call this $R(\omega)$ the repeller corresponding to $A(\omega)$ with respect to $U(\omega)$.)

Proof. The proof is similar to that of Lemma 4.1 in [14]. By the forward invariance of $U(\omega)$, we have

$$
\varphi\left(t, \theta_{-t} \omega\right) U\left(\theta_{-t} \omega\right) \subset \varphi\left(s, \theta_{-s} \omega\right) U\left(\theta_{-s} \omega\right), \text { for } \forall t \geq s, s, t \in \mathbb{R}
$$

Hence we have

$$
\begin{aligned}
A(\omega) & =\bigcap_{T \geq 0} \overline{\bigcup_{t \geq T} \phi\left(t, \theta_{-t} \omega\right) U\left(\theta_{-t} \omega\right)}=\bigcap_{n \in \mathbb{N}} \varphi\left(n, \theta_{-n} \omega\right) \overline{U\left(\theta_{-n} \omega\right)} \\
& =\lim _{n \rightarrow \infty} \varphi\left(n, \theta_{-n} \omega\right) \overline{U\left(\theta_{-n} \omega\right)}
\end{aligned}
$$

and

$$
\begin{aligned}
B(A, U)(\omega) & =\bigcup_{t \geq 0} \varphi\left(-t, \theta_{t} \omega\right) U\left(\theta_{t} \omega\right)=\bigcup_{n \in \mathbb{N}} \varphi\left(-n, \theta_{n} \omega\right) U\left(\theta_{n} \omega\right) \\
& =\lim _{n \rightarrow \infty} \varphi\left(-n, \theta_{n} \omega\right) U\left(\theta_{n} \omega\right) .
\end{aligned}
$$

Since $U(\omega)$ is a forward invariant random open set, we have that $V(\omega):=X-\overline{U(\omega)}$ is a backward invariant random open set (see page 35 of [1]). Denote $\tilde{R}(\omega):=\alpha_{V}(\omega)$, then $\tilde{R}(\omega)$ is a random repeller with a repelling neighborhood $V(\omega)$. By the definition of alphalimit set, we have $R(\omega) \subset \tilde{R}(\omega)$ due to the fact $R(\omega) \subset V(\omega)$ and the invariance of $R(\omega)$. If there exists some $x_{0} \in \tilde{R}(\omega) \backslash R(\omega)$, then $x_{0} \in B(A, U)(\omega)$. Therefore there exists some $t_{0} \geq 0$ such that $\varphi\left(t_{0}, \omega\right) x_{0} \in U\left(\theta_{t_{0}} \omega\right)$. Noticing that $\tilde{R}(\omega)$ is an invariant random closed set, we have $\varphi\left(t_{0}, \omega\right) x_{0} \in \tilde{R}\left(\theta_{t_{0}} \omega\right)$. This is a contradiction to the fact $\tilde{R}(\omega) \cap U(\omega)=\emptyset$ for each $\omega$. Therefore we obtain $R(\omega)=\tilde{R}(\omega)$, i.e. $R(\omega)$ is a repeller with a repelling neighborhood $V(\omega)$. Now we will show $B(R, V)(\omega)=X-A(\omega)$. In fact

$$
\begin{aligned}
B(R, V)(\omega) & =\bigcup_{n \in \mathbb{N}} \varphi\left(n, \theta_{-n} \omega\right) V\left(\theta_{-n} \omega\right) \\
& =\lim _{n \rightarrow \infty} \varphi\left(n, \theta_{-n} \omega\right) V\left(\theta_{-n} \omega\right) \\
& =\lim _{n \rightarrow \infty} \varphi\left(n, \theta_{-n} \omega\right)\left[X-\overline{U\left(\theta_{-n} \omega\right)}\right] \\
& =\lim _{n \rightarrow \infty}\left[X-\varphi\left(n, \theta_{-n} \omega\right) \overline{U\left(\theta_{-n} \omega\right)}\right]
\end{aligned}
$$




$$
=X-A(\omega),
$$

where (9) and (10) hold completely similar to (8) if we take $t=-t$, (11) follows from the fact that $\varphi(n, \omega)$ is a homeomorphism on $X$ and (12) holds by (7). This completes the proof of the lemma.

Similar to the proofs of Lemmas 3.4, 3.6 and 3.7 in [13], we can obtain the following result (Notice that in these proofs, the compactness is only relevant in proving Lemma 3.4, where we use compactness to conclude $\bar{d}(\omega)>0$. In Definitions 5.1 and 5.2, by requesting that the neighborhood of attractor be absorbing we overcome this difficulty when $X$ loses compactness.):

Theorem 5.1. Assume $X$ is a Polish space, $U(\omega)$ is an absorbing set, $A(\omega)$ is the random local attractor determined by $U(\omega)$, and $B(A, U)(\omega)$ is the basin of $A(\omega)$ with respect to $U(\omega)$, then

$$
X-\mathcal{C R}_{\varphi}(\omega)=\bigcup[B(A, U)(\omega)-A(\omega)]
$$

almost surely, where the union is taken over all absorbing sets.

Lemma 5.2. Assume $A(\omega)$ is an attractor with an absorbing neighborhood $U(\omega)$ which determines $A(\omega)$. Then there is a forward invariant absorbing neighborhood $\tilde{U}(\omega)$ of $A(\omega)$ such that $\Omega_{\tilde{U}}(\omega)=A(\omega)$.

Proof. Noting that the compactness is not required when we prove $\Omega_{\tilde{U}}(\omega)=\Omega_{U}(\omega)=$ $A(\omega)$ in Lemma 3.1. Hence there exists a forward invariant neighborhood $\tilde{U}(\omega)$ of $A(\omega)$ such that $\Omega_{\tilde{U}}(\omega)=A(\omega)$ when $X$ loses compactness. We only need to show that $\tilde{U}(\omega)$ is absorbing. Assume that there exist $\epsilon(\omega), T(\omega)>0$ such that

$$
B_{\epsilon}\left(U_{T}(\omega)\right) \subset U(\omega) .
$$

By the fact $\Omega_{\tilde{U}}(\omega)=\Omega_{U}(\omega)=A(\omega)$ we obtain that there exists $t=t(\omega)$ such that

$$
\tilde{U}_{t}(\omega) \subset U_{T}(\omega)
$$

where $\tilde{U}_{t}(\omega)$ is defined similar to $U_{T}(\omega)$. Define

$$
\tilde{T}(\omega):=\inf \left\{t \in \mathbb{R}^{+} \mid \varphi\left(t, \theta_{-t} \omega\right) \overline{\tilde{U}\left(\theta_{-t} \omega\right)} \subset U_{T}(\omega)\right\}
$$

then $\tilde{T}(\omega)$ is measurable by similar argument to the proof of Lemma 3.5 in [13. Thus $B_{\epsilon}\left(\tilde{U}_{\tilde{T}}(\omega)\right) \subset U(\omega) \subset \tilde{U}(\omega)$, and hence $\tilde{U}(\omega)$ is absorbing. The proof is complete.

Similar to the case when $X$ is compact, we have the following theorem:

Theorem 5.2. Assume $X$ is a Polish space, $U(\omega)$ is a forward invariant absorbing set, $A(\omega)$ is the random local attractor determined by $U(\omega)$ and $R(\omega)$ is the repeller corresponding to $A(\omega)$ with respect to $U(\omega)$, then

$$
\mathcal{C R}_{\varphi}(\omega)=\bigcap[A(\omega) \cup R(\omega)]
$$

almost surely, where the intersection is taken over all forward invariant absorbing sets.

Proof. By Theorem 5.1, we only need to verify

$$
\bigcap_{U \in \mathcal{U}}[A(\omega) \cup R(\omega)] \subset \mathcal{C} \mathcal{R}_{\varphi}(\omega)
$$


where $\mathcal{U}$ denotes the set of all forward invariant absorbing sets. To this end, we only need to show

$$
\bigcup[B(A, U)(\omega)-A(\omega)] \subset \bigcup_{U \in \mathcal{U}}[B(A, U)(\omega)-A(\omega)],
$$

where the union on the left hand is taken over all absorbing sets. By Lemma 5.2 we know that for an attractor $A(\omega)$ with an absorbing neighborhood $U(\omega)$, there is an absorbing neighborhood $\tilde{U}(\omega) \in \mathcal{U}$ of $A(\omega)$ such that $\Omega_{\tilde{U}}(\omega)=\Omega_{U}(\omega)=A(\omega)$ and $U(\omega) \subset \tilde{U}(\omega)$. Thus we have

$$
B(A, U)(\omega) \subset B(A, \tilde{U})(\omega) .
$$

Therefore we have proved (14) and hence the theorem.

By mimicking the proof when the state space is compact, we can obtain the complete Lyapunov function for $\varphi$ when $X$ is not compact; furthermore, we can discuss the chain transitive components etc completely similar to the compact case. In fact, the compactness is not relevant during these steps. Hence we omit details here. Therefore we have the random Conley decomposition theorem on Polish spaces:

Theorem 5.3. (random Conley decomposition theorem on Polish spaces). Any random dynamical system (with a separable metric space endowed with a probability measure as base space) on a Polish space decomposes the Polish space into a random chain recurrent part and a random gradient-like part.

Example 5.1. Consider the Lorenz system in $\mathbb{R}^{3}$ described by the equations:

$$
\left\{\begin{array}{l}
\dot{x}=\sigma(y-x), \\
\dot{y}=\rho x-y-x z, \\
\dot{z}=x y-\beta z
\end{array}\right.
$$

with parameters $\sigma, \rho, \beta>0$. Assume that the parameter $\sigma, \rho, \beta$ are perturbed by real noises, i.e.

$$
\begin{aligned}
\sigma(\omega) & =\sigma+\xi(\omega), \\
\rho(\omega) & =\rho+\eta(\omega), \\
\beta(\omega) & =\beta+\zeta(\omega) .
\end{aligned}
$$

Assume that the perturbed parameters are still positive and $\rho(\omega)<\sigma(\omega) \leq 1$ almost surely. Then the perturbed random ODE generates an RDS and we denote it by $\varphi$, see [1] for details. Let

$$
L(\omega, x, y, z)=x^{2}+y^{2}+z^{2}
$$

and define the random set

$$
D_{r}(\omega):=\{(x, y, z) \mid L(\omega, x, y, z) \leq r\}
$$

Then clearly $D_{r}(\omega)$ is a random compact set. Moreover, the derivative of $L$ with respect to $t$ along the orbits of $\varphi$

$$
\begin{aligned}
\frac{\mathrm{d} L}{\mathrm{~d} t} & =2 x(\sigma(y-x))+2 y(\rho x-y-x z)+2 z(x y-\beta z) \\
& =-2 \sigma x^{2}-2 y^{2}-2 \beta z^{2}+2(\rho+\sigma) x y \\
& \leq-(\sigma-\rho) x^{2}-(2-\rho-\sigma) y^{2}-2 \beta z^{2}
\end{aligned}
$$


whenever $(x, y, z) \neq(0,0,0)$ by the assumption $\rho(\omega)<\sigma(\omega) \leq 1$, where $\rho=\rho\left(\theta_{t} \omega\right)$, $\sigma=\sigma\left(\theta_{t} \omega\right), \beta=\beta\left(\theta_{t} \omega\right)$ and $X=X(t, \omega)$ with $X:=(x, y, z)$. Therefore, for $\forall X \in D_{r}(\omega)$ and $\forall t>0$ we have $L\left(\theta_{t} \omega, \varphi(t, \omega) X\right)<L(\omega, X) \leq r$, i.e. $\varphi(t, \omega) X \in D_{r}\left(\theta_{t} \omega\right)$. Hence $D_{r}(\omega)$ is a forward invariant random compact set and clearly it determines a random attractor $A(\omega)=\{0\}$ with the basin $B(A)(\omega)=\mathbb{R}^{3}$ almost surely. And by (15) we easily know that $A(\omega)$ is the only non-trivial attractor of $\varphi$. Therefore, by Theorem 5.2 we obtain that the random chain recurrent set is $\{0\}$ and hence it is the only random chain transitive component.

Notes to [13]: The definition of random open set in [13] should be same as Definition 2.1 of present paper. Correspondingly, the items (i), (ii) and (vi) of Proposition 2.1 in 13 should be stated as follows:

(i) $D(\omega)$ is a random closed set in $X$ if and only if the set $\{\omega: D(\omega) \cap U \neq \emptyset\}$ is measurable for any open set $U \subset X$;

(ii) $D(\omega)$ is measurable if and only if $\overline{D(\omega)}$ is a random closed set;

(vi) If $\left\{D_{n}, n \in \mathbb{N}\right\}$ is a sequence of random open sets and there exists $n_{0} \in \mathbb{N}$ such that $D_{n_{0}}^{c}$ is a random compact set, then $D=\bigcup_{n \in \mathbb{N}} D_{n}$ is also a random open set. Or if $\left\{D_{n}, n \in \mathbb{N}\right\}$ is a sequence of measurable multifunctions, then $D=\bigcup_{n \in \mathbb{N}} D_{n}$ is also a measurable multifunction.

By Lemma 3.1 and Remark 3.1 of present paper, the proofs of Lemmas 3.1, 3.3 and 3.6 in [13] can be greatly simplified and some minor errors can be easily avoided.

\section{Acknowledgements}

The author expresses his sincere thanks to Professor Yong Li for his instructions and many invaluable suggestions. The author is very grateful to Professors Youqing Ji and Xiaoyun Yang as well as Shuguan Ji and Menglong Su for helpful discussions. Many thanks to the anonymous referees for their careful reading the manuscript and invaluable comments which greatly improved the presentation of the paper.

\section{References}

[1] Arnold L 1998 Random Dynamical Systems (Berlin: Springer)

[2] Arnold L and Schmalfuss B 2001 Lyapunov's second method for random dynamical systems J. Diff. Eqns 177 235-65

[3] Bhatia N P and Szegö G P 1970 Stability Theory of Dynamical Systems (Berlin: Springer)

[4] Castaing C and Valadier M 1977 Convex Analysis and Measurable Multifunctions (Lecture Notes in Mathematics vol 580) (Berlin: Springer)

[5] Chueshov I 2002 Monotone Random Systems Theory and Applications (Lecture Notes in Mathematics vol 1779) (Berlin: Springer) 
[6] Conley C 1978 Isolated Invariant Sets and the Morse Index (Conf. Board Math. Sci. vol 38) (Providence, RI: American Mathematical Society)

[7] Crauel H, Duc L H and Siegmund S 2004 Towards a Morse theory for random dynamical systems Stoch. Dyn. 4 277-96

[8] Crauel H and Flandoli F 1994 Attractors for random dynamical systems Probab. Theory Relat. Fields 100 365-93

[9] Franks J 1988 A variation of Poincaré-Birkhoff theorem Hamiltonian Dynamical Systems (Contemporary Mathematics vol 81) (Providence, RI: American Mathematical Society) pp 111-7

[10] Hurley M 1991 Chain recurrence and attraction in non-compact spaces Ergod. Theory Dyn. Syst. 11 709-29

[11] Hurley M 1992 Noncompact chain recurrence and attraction Proc. Am. Math. Soc. 115 1139-48

[12] Hurley M 1995 Chain recurrence, semiflows, and gradients J. Dyn. Diff. Eqns. 7 $437-56$

[13] Liu Z 2006 The random case of Conley's theorem Nonlinearity 19 277-91

[14] Liu Z, Ji S and Su M 2006 Attractor-repeller pair, Morse decomposition and Lyapunov function for random dynamical systems http://arxiv.org/abs/math.DS/0606205

[15] Norton D E 1995 The fundamental theorem of dynamical systems Comment. Math. Univ. Carolin. 36 585-97 